\title{
Research on Wideband Differential-Mode Current Injection Testing Technique Based on Directional Coupling Device
}

\author{
Xiaodong Pan, Guanghui Wei, Xinfu Lu, Lisi Fan, and Xing Zhou \\ National Key Laboratory of Electromagnetic Environment Effects, Mechanical Engineering College, \\ Heping West Road, Shijiazhuang 050003, China \\ Correspondence should be addressed to Xiaodong Pan; panxiaodong1980@sina.com
}

Received 7 April 2014; Accepted 23 June 2014; Published 15 July 2014

Academic Editor: Wenhua Yu

Copyright (c) 2014 Xiaodong Pan et al. This is an open access article distributed under the Creative Commons Attribution License, which permits unrestricted use, distribution, and reproduction in any medium, provided the original work is properly cited.

This paper presents a new kind of differential-mode current injection test method. The equal response voltage on the cable or the antenna port of the equipment under test (EUT) is regarded as equivalent principle for radiation and injection test. The injection and radiation response analysis model and the injection voltage source extrapolation model in high intensity radiated field are established. The conditions of using differential-mode current injection as a substitute for radiation are confirmed. On the basis of the theoretical analysis, the function and structure design scheme of the directional coupling device is proposed. The implementation techniques for the single differential-mode current injection method (SDMCI) and the double differential-mode current injection method (DDMCI) are discussed in detail. The typical nonlinear response interconnected systems are selected as the EUT. The test results verify the validity of the SDMCI and DDMCI test methods.

\section{Introduction}

Bulk current injection (BCI) is a kind of traditional EMC test method. Essentially, the interference current is injected into the cable of the equipment to substitute for radiation susceptibility test $[1,2]$. As a kind of complementary method, the core of the BCI research is how to keep the equivalence with the radiation test method in broader application range and higher precision [3-6]. Although the traditional BCI method has been proposed for nearly half a century, there are still insufficiencies to substitute for the high intensity radiated field (HIRF) effects test. First, the application frequency range is limited. When the test frequency becomes higher, the injection and monitoring currents change to be sensitive to the position of the cable because of the standing wave, and the performance of the ferrite current probe descends severely. These factors make the testing precision and injection efficiency decline obviously [7-9]. Numerous studies show that when the test frequency is higher than $400 \mathrm{MHz}$, the present BCI method cannot satisfy the practical requirements $[10,11]$. Second, the BCI method cannot accurately substitute for
HIRF radiation effect test for nonlinear systems. At present, the BCI method is effective when the relation between the radiated field intensity and the induced current on the cable of the equipment is linear. Thus, the injection current substituted for HIRF could be extrapolated according to the linear corresponding relation. But the majority of equipment under the condition of HIRF is nonlinear. If the same test method is applied for the nonlinear system, it may cause considerable error because of dissatisfying the extrapolation condition. Third, the BCI method is a common-mode current injection test method. It means that the interference signal injected by the current probe is a common-mode signal [12-14]. This method cannot simulate the effects caused by the differential-mode interference signal received from the antenna. The application range of the BCI method is limited.

In conclusion, HIRF in large-scale test space is very difficult to simulate under the condition of laboratory. Meanwhile, there are still many insufficiencies for the traditional BCI method to carry out the injection susceptibility tests. Hence, our research team proposes a new kind of wideband 


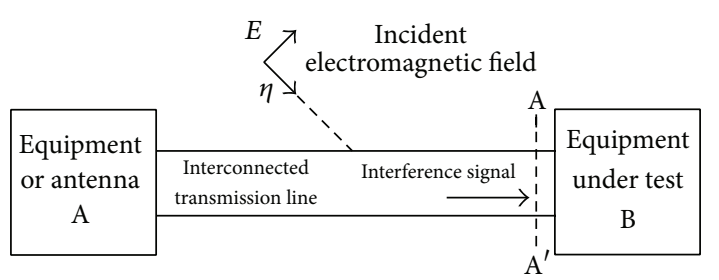

Figure 1: The structure of a typical interconnected system.

differential-mode current injection test method for system level EMC test.

\section{Theoretical Analysis Model}

The theoretical equivalent principle between the injection and radiation test method is the equal response of the equipment [15-17]. The engineering equivalent principle is the same effects caused by the two test methods. If the response voltage or the induced current on the cable port of the equipment could be ensured to be equal, the equivalence of the two test methods can be achieved $[18,19]$. In this paper, the equal response voltage on the cable port of the equipment is selected as the equivalent principle of the two test methods finally.

2.1. Equivalence Analysis Model between the Injection and Radiation Response. In this paper, the typical interconnected system is composed of two types of equipment and the interconnected cable. It is shown in Figure 1. It is assumed that equipment $\mathrm{B}$ is the EUT and equipment $\mathrm{A}$ is either the interconnected equipment or the receiving antenna. In order to calculate the radiation response voltage of the equipment $\mathrm{B}$, the interconnected system is divided into two parts at the position of $\mathrm{A}-\mathrm{A}^{\prime}$ reference plane. $\mathrm{A}-\mathrm{A}^{\prime}$ is located at the input port of the equipment B. The left branch of $A-A^{\prime}$ can be equivalent to the Thevenin equivalent circuit. It is shown in Figure 2(a), where $Z_{\mathrm{SR}}$ is the input impedance of the left branch of $\mathrm{A}-\mathrm{A}^{\prime}$ and $U_{\mathrm{SR}}$ is the open-circuit voltage.

According to the transmission line theory, the input impedance $Z_{\mathrm{SR}}$ can be calculated as follows:

$$
Z_{\mathrm{SR}}=Z_{\mathrm{C}} \frac{1+\Gamma_{\mathrm{A}} e^{-j 2 \gamma l}}{1-\Gamma_{\mathrm{A}} e^{-j 2 \gamma l}},
$$

where $\Gamma_{\mathrm{A}}$ is the reflection coefficient of the equipment $\mathrm{A}, Z_{\mathrm{C}}$ is the characteristic impedance of the transmission line, $l$ is the transmission line length between $\mathrm{A}-\mathrm{A}^{\prime}$ and the equipment $\mathrm{A}$, and $\gamma$ is the propagation constant.

The open-circuit voltage $U_{\mathrm{SR}}$ can be calculated with the BLT equation [20-22]. Assuming that $A-A^{\prime}$ port is open, that is, $\Gamma_{\mathrm{B}}=1$, the open-circuit voltage $U_{\mathrm{SR}}$ caused by the transmission line coupling can be calculated as follows:

$$
U_{\mathrm{SR}}=\frac{2}{1-\Gamma_{\mathrm{A}} e^{-2 \gamma l}}\left(e^{-\gamma l} S_{1}+\Gamma_{\mathrm{A}} e^{-2 \gamma l} S_{2}\right),
$$

where $S_{1}$ and $S_{2}$ are the source parameters in the BLT equation. If the equipment $\mathrm{A}$ is a receiving antenna, it can be regarded as a lumped voltage source $U_{0}$. The open-circuit voltage $U_{\mathrm{SR}}$ caused by $U_{0}$ can be calculated as follows:

$$
U_{\mathrm{SR}}=U_{0}\left(1-\Gamma_{\mathrm{A}} e^{-2 \gamma l}\right)^{-1} e^{-\gamma l}\left(1-\Gamma_{\mathrm{A}}\right)
$$

In (2) and (3), there is a linear relation between $S_{1}, S_{2}$, $U_{0}$, and the radiated electric field intensity $E[20,21]$. We define that the linear transfer function between $U_{\mathrm{SR}}$ and $E$ is $f$. Hence, the open-circuit voltage $U_{\text {SR }}$ can be simplified as follows:

$$
U_{\mathrm{SR}}=f(E) .
$$

Therefore, in Figure 2(a), the radiation response $U_{\mathrm{BR}}$ on the impedance $Z_{\mathrm{B}}$ can be derived as follows:

$$
U_{\mathrm{BR}}=\frac{Z_{\mathrm{B}}}{Z_{\mathrm{SR}}+Z_{\mathrm{B}}} U_{\mathrm{SR}}=\frac{Z_{\mathrm{B}}}{Z_{\mathrm{SR}}+Z_{\mathrm{B}}} f(E) .
$$

According to the above radiation analysis process, under the condition of the injection test, the equivalent circuit can be easily obtained. It is shown in Figure 2(b), where $U_{\mathrm{SI}}$ is the injection voltage source, $Z_{\mathrm{SI}}$ is the equivalent impedance, and $U_{\mathrm{BI}}$ is the injection response of equipment $\mathrm{B}$. The injection response $U_{\mathrm{BI}}$ can be derived as follows:

$$
U_{\mathrm{BI}}=\frac{Z_{\mathrm{B}}}{Z_{\mathrm{SI}}+Z_{\mathrm{B}}} U_{\mathrm{SI}} \text {. }
$$

According to the equivalent principle of the two test methods, that is, $U_{\mathrm{BI}}=U_{\mathrm{BR}}$, the equivalent injection voltage source $U_{\mathrm{SI}}$ can be derived as follows:

$$
U_{\mathrm{SI}}=\frac{Z_{\mathrm{SI}}+Z_{\mathrm{B}}}{Z_{\mathrm{SR}}+Z_{\mathrm{B}}} U_{\mathrm{SR}}=\frac{Z_{\mathrm{SI}}+Z_{\mathrm{B}}}{Z_{\mathrm{SR}}+Z_{\mathrm{B}}} f(E) .
$$

Equation (7) confirms the equivalent relation between injection voltage $U_{\text {SI }}$ and radiation electric field intensity $E$ theoretically, which guarantees the equivalence between the injection and the radiation effects test.

2.2. Injection Voltage Source Linear Extrapolation Model. Wideband differential-mode current injection testing technique proposed in this paper is used to substitute for high intensity radiated effects test. The crucial question is how to acquire the equivalent injection voltage source $V_{\mathrm{SI}}$. According to the above equivalent ideas, we plan to acquire $V_{\mathrm{SI}}$ by extrapolating $U_{\text {SI }}$ which is used to substitute for low intensity radiated effects test. Generally speaking, the majority of systems are nonlinear systems under the condition of HIRF. It means that the response voltage on the system ports will not keep linear relation with the radiated field. Thus, for nonlinear systems, how to extrapolate the injection voltage source $U_{\mathrm{SI}}$ is the crucial problem.

As we know, the electromagnetic radiation effects on equipment mainly include interference, degradation, failure, and damage. On the basis of theoretical analysis and experimental research for typical nonlinear systems, the action process of electromagnetic radiated energy can be divided into two subprocesses. One is the field to wire coupling 


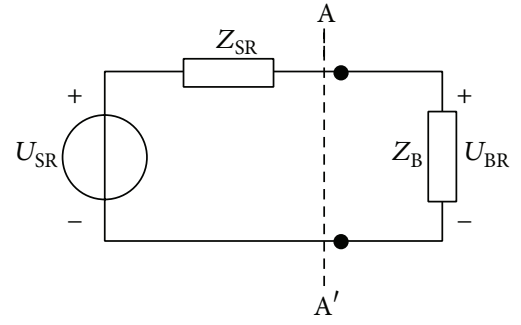

(a)

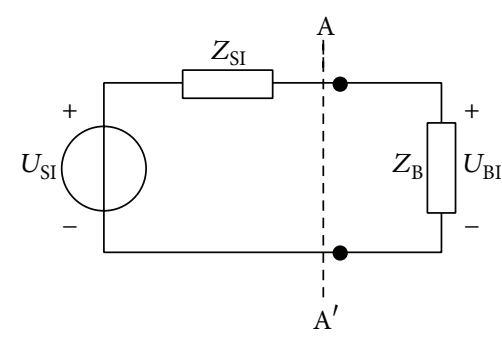

(b)

FIGURE 2: The equivalent circuit model for analyzing the equipment B response. (a) Radiation test. (b) Injection test.

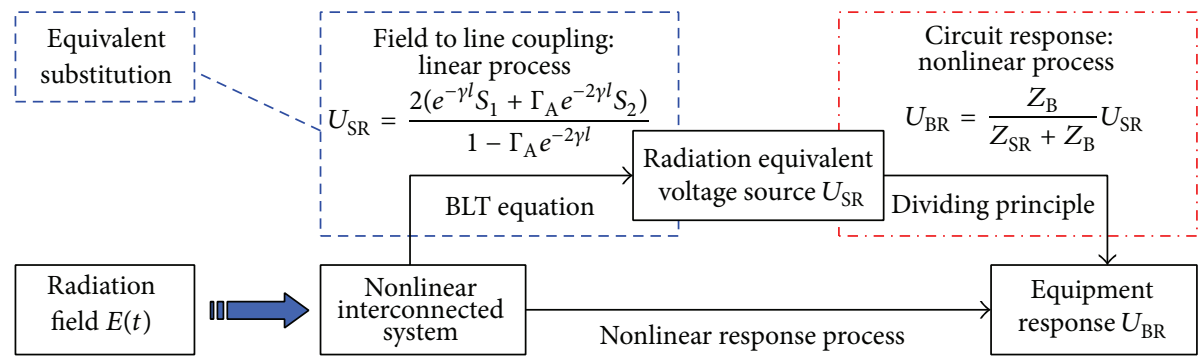

FIGURE 3: Radiation response process for nonlinear interconnected system.

process, and the other is the circuit response process of the module and device. The radiation response process is shown in Figure 3.

According to the electromagnetic field theory, the field to wire coupling is a linear process, and the circuit response of the module and device is a nonlinear process. If the excitation effects of the injection source $U_{\mathrm{SI}}$ and the lumped radiation source $U_{\mathrm{SR}}$ can be guaranteed equal at the input port of the module and device, the nonlinear circuit response in the injection experiment will be the same as in the radiation experiment. Under the condition of HIRF, in order to ensure the effect excited by injection source is the same as excited by the lumped radiation source, according to (5) and (6), two conditions must be satisfied. One is the same open-circuit voltage. The other is the same voltage division rate in the radiation and the injection test.

For the reason that $U_{\mathrm{SR}}$ is the equivalent lumped voltage source obtained in the linear process of the field to wire coupling or antenna receiving, the injection voltage source $V_{\text {SI }}$ applied to substitute for HIRF radiation test can be obtained by linearly extrapolating from $U_{\mathrm{SI}}$. The $U_{\mathrm{SI}}$ is used to substitute for the low intensity radiated field effects test; $U_{\mathrm{SI}}=$ $U_{\mathrm{SR}}$. So there is no problem to satisfy the first condition. For the second condition, in order to guarantee the same voltage division rate under the condition of different excitation intensity, it is required that the output impedance of the injection voltage source is equal to the lumped radiation voltage source; that is, $Z_{\mathrm{SI}}=Z_{\mathrm{SR}}$. When the above two conditions can be satisfied, the equivalence between the differential-mode current injection test and HIRF radiation effect test will be guaranteed definitely.

\section{Design for Supporting Equipment}

Directional coupling device (DCD) is the supporting equipment for the wideband differential-mode current injection test. The typical connection mode is shown in Figure 4. On the premise of normal working status for the interconnected system, the differential-mode current injection test for equipment B can be carried out via the injection port of the DCD.

In order to satisfy the requirements of substituting the differential-mode current injection test for the radiation test, the DCD should contain the following ports. First, the DCD should contain the pass-through ports which are used to transmit the working signal between the interconnected equipment. It requires that the insertion loss is smaller than $0.5 \mathrm{~dB}$. Second, the device should contain the injection port which is used to inject the differential-mode interference signal to the equipment $\mathrm{B}$. It requires that the frequency band of the injection port is sufficient and the injection signal coupled into the equipment B is distortion-free. Third, the device should contain the monitoring port which is used to monitor the forward voltage signal on the transmission line of the interconnected system. It also requires that the frequency band is sufficient, and the monitoring signal is distortion-free.

According to the above function requirements of the $\mathrm{DCD}$, the design scheme based on the directional coupler theory is confirmed. The DCD can be composed of two directional couplers. It is shown in Figure 5. From port 1 to port 2 of the DCD is the pass-through channel. It is used to transmit the working signal between the equipment $\mathrm{A}$ and equipment B. Port 4 is the injection port. It is used to inject the differential-mode interference signal to equipment B. Port 


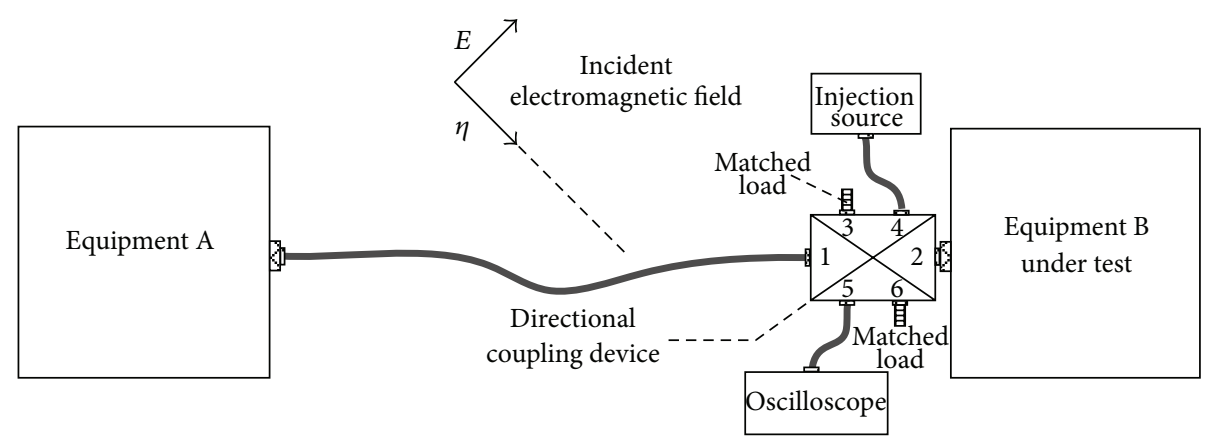

FIGURE 4: The sketch map of connection mode for DCD.

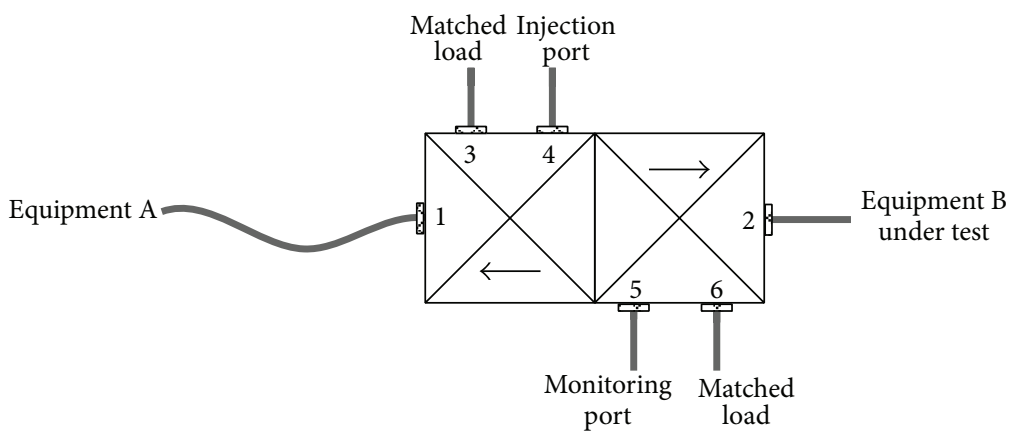

Figure 5: The function and structure of the DCD.

5 is the monitoring port. It is used to monitor the forward voltage signal on the transmission line of the interconnected system. Port 3 and port 6 are the isolation ports. They will be connected with the matched loads when the DCD is in use.

The above six ports DCD can be regarded as lossless and reciprocal network. The scattering matrix $\mathbf{S}$ of the DCD satisfies the characteristic of symmetry and unitarity; that is, $\mathbf{S}^{\mathrm{T}}=\mathbf{S}$ and $\mathbf{S}^{\mathrm{T}} \mathbf{S}^{*}=1$. According to the technology requirements of the DCD, the insertion loss between port 1 and port 2 is not bigger than $0.5 \mathrm{~dB}$. So the parameters $S_{21}$ and $S_{12}$ satisfy the equation as follows:

$$
S_{21}=S_{12} \geq 0.944 \text {. }
$$

Port 4 is the injection port. It is used to inject differentialmode interference signal to equipment $B$. It requires higher injection efficiency, so the coupling coefficient should be as small as possible. However, too small coupling coefficient cannot satisfy the insertion loss requirement between port 1 and port 2; that is, $S_{21}=S_{12} \geq 0.944$. Taking all these into account, the coupling coefficient of port 3 and port 4 is selected as $10 \mathrm{~dB}$.

According to the transmission characteristic of the traditional symmetrical directional coupler, the phase shift between the coupling channel signal and the main channel signal is $90^{\circ}$. If the pulse signal transmits in the main channel, the output signal waveform of the coupling port will change obviously [23]. Thus, the antisymmetry directional coupler design scheme is adopted in this paper. It makes the phase shift between the forward coupling signal and the main channel signal be $0^{\circ}$ and the phase shift between the backward coupling signal and the main channel signal be $180^{\circ}$. It is assumed that port 4 is the forward coupling port of the main channel from port 2 to port 1 and port 5 is the forward coupling port of the main channel from port 1 to port 2. The directions of the two arrows in Figure 5 show the forward direction of the two antisymmetry directional couplers separately. Assuming that the $S_{21}$ parameter is a positive real number, the $S_{31}$ parameter is a negative real number. If the coupling coefficient of port 3 and port 4 is $10 \mathrm{~dB}$, it can be derived that

$$
S_{31}=-\sqrt{0.1}
$$

According to the unitarity of the scattering matrix $\mathbf{S}$, considering the insert loss requirement of $S_{21} \geq 0.944$, it can be derived as follows:

$$
\left|S_{51}\right| \leq 0.094
$$

For the phase shift between the forward coupling signal and the main channel signal which is $0^{\circ}$, considering the energy coupling action of port 3 , the parameter $S_{51}$ can be derived as follows:

$$
S_{51}=\sqrt{(1-0.1) 10^{-x / 10}}
$$

where $x$ is the coupling coefficient of port 5 and port 6 . Considering the manufacturing technology and the convenience for application, the coupling coefficient of port 5 and port 6 is confirmed to be $20 \mathrm{~dB}$. 


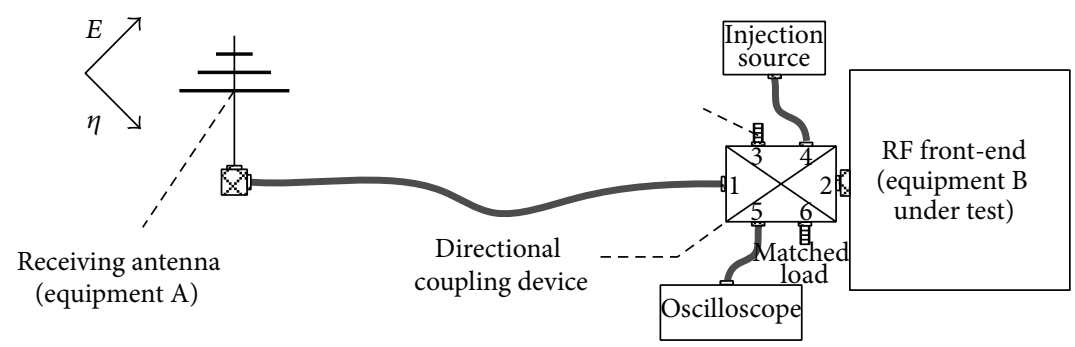

FIGURE 6: The typical test configuration of SDMCI test method.

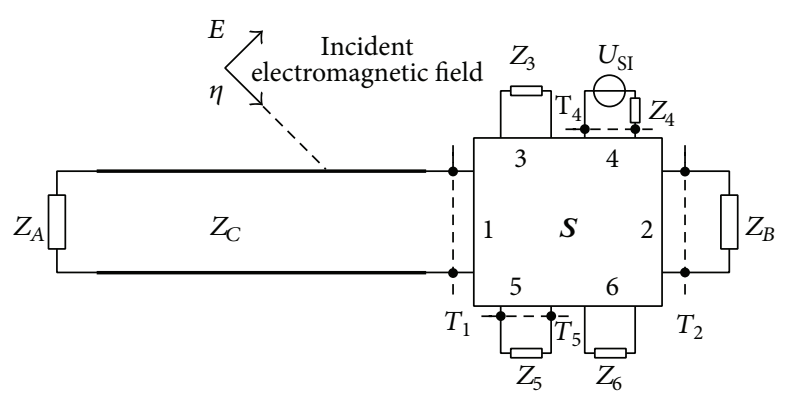

FIGURE 7: The equivalent circuit model when the DCD is inserted into the interconnected system.

From the above analysis process, the scattering matrix $\mathbf{S}$ of the six ports DCD can be calculated as follows:

$$
\mathbf{S}=\left[\begin{array}{cccccc}
0 & 0.944 & -0.316 & 0 & 0.095 & 0 \\
0.944 & 0 & 0 & 0.315 & 0 & -0.1 \\
-0.316 & 0 & 0 & 0.949 & 0 & 0 \\
0 & 0.315 & 0.949 & 0 & 0.032 & 0 \\
0.095 & 0 & 0 & 0.032 & 0 & 0.995 \\
0 & -0.1 & 0 & 0 & 0.995 & 0
\end{array}\right]
$$

\section{Implementation Technology of SDMCI}

Because of the directional injection characteristic of the DCD, the single differential-mode current injection (SDMCI) method is used to carry out injection test only for one end equipment in the interconnected system. The typical application is to carry out differential-mode current injection test for antenna receiving system. The test configuration of the SDMCI is shown in Figure 6. It can simulate the effects caused by the interference signal received from the antenna when the antenna receiving system is in the normal working condition.

\subsection{Equivalent Condition Analysis When DCD Is Inserted} into the Interconnected System. The forward voltage signal extraction, interference signal injection, and normal transmission signal monitoring are realized with the support of the DCD. However, whether it satisfies the equivalent condition discussed above or not is the crucial problem, which is analyzed as follows.

The DCD can be equivalent to six ports black box network. The equivalent circuit model is shown in Figure 7, where $T_{1}, T_{2}, T_{4}$, and $T_{5}$ are the reference plane at different ports of the DCD, $Z_{3}$ and $Z_{6}$ are the matched load, $Z_{5}$ is the input impedance of the oscillograph, $U_{\mathrm{SI}}$ is the open-circuit voltage of the injection voltage source, and $Z_{4}$ is the internal impedance of the injection voltage source.

As for the radiation test, the left part of the reference plane $T_{1}$ can be equivalent to the source wave $\widehat{a}_{1 \mathrm{R}}$ and the reflection coefficient $\Gamma_{1}$. It also can be equivalent to the open-circuit voltage $U_{\mathrm{SR}}$ and the source impedance $Z_{1}$. Similarly, as for the differential-mode current injection test, the top part of reference plane $T_{4}$ can be equivalent to the source wave $\widehat{a}_{4 \mathrm{I}}$ and the reflection coefficient $\Gamma_{4}$. It also can be equivalent to the open-circuit voltage $U_{\text {SI }}$ and the source impedance $Z_{4}$. Therefore, the equivalent circuit model for the radiation and injection test is shown in Figure 8. It can be seen that the network model without source is the same in the radiation and injection test. The difference is that the equivalent source is located in the different ports of the DCD.

On the basis of the equivalent source wave theory in microwave engineering [24], under the condition of the radiation and injection test, the relation between the equivalent source wave and equivalent voltage source is as follows:

$$
\begin{gathered}
\widehat{a}_{1 \mathrm{R}}=\frac{U_{\mathrm{SR}}\left(1-\Gamma_{1}\right)}{2 \sqrt{Z_{\mathrm{C}}}}, \\
\widehat{a}_{4 \mathrm{I}}=\frac{U_{\mathrm{SI}}\left(1-\Gamma_{4}\right)}{2 \sqrt{Z_{\mathrm{C}}}}=\frac{U_{S I}}{2 \sqrt{Z_{\mathrm{C}}}} .
\end{gathered}
$$

In order to analyze the response of the equipment $\mathrm{B}$, the left part of the reference plane $T_{2}$ is required to simplify further. In the radiation test, the left part of $T_{2}$ is equivalent to the source wave $\widehat{b}_{2 \mathrm{R}}$ and the reflection coefficient $\Gamma_{2 \mathrm{R}}^{\prime}$. In the injection test, the left part of $T_{2}$ is equivalent to the source wave $\widehat{b}_{2 \mathrm{I}}$ and the reflection coefficient $\Gamma_{2 \mathrm{I}}^{\prime}$. It is shown in Figure 9. 


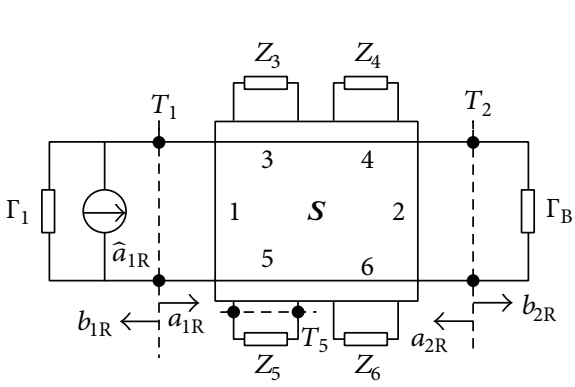

(a)

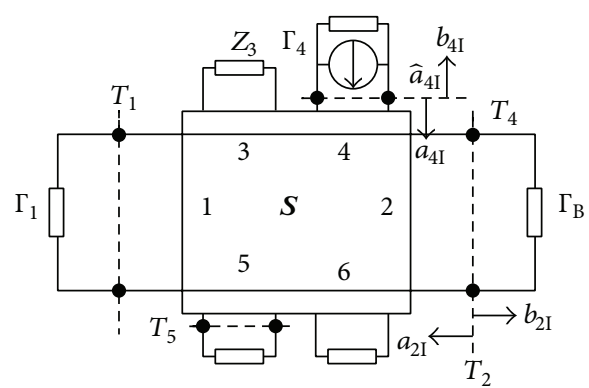

(b)

FIGURE 8: The equivalent circuit model for (a) radiation test and (b) injection test.

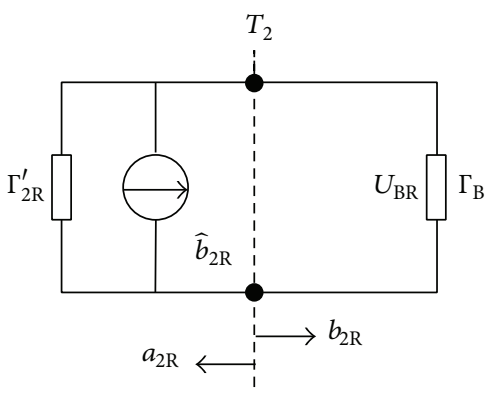

(a)

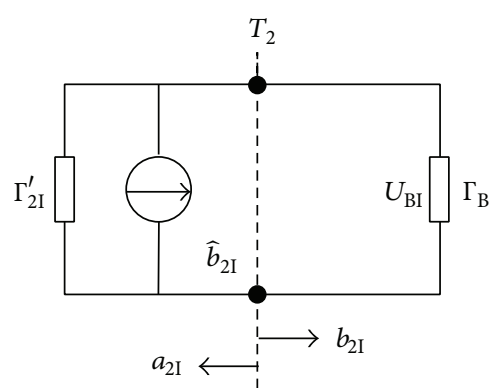

(b)

FIGURE 9: The further simplified circuit models for (a) radiation test and (b) injection test.

According to the equivalent source wave theorem [24], $\widehat{b}_{2 \mathrm{R}}, \widehat{b}_{2 \mathrm{I}}, \Gamma_{2 \mathrm{R}}^{\prime}$, and $\Gamma_{2 \mathrm{I}}^{\prime}$ can be derived as follows:

$$
\begin{gathered}
\widehat{b}_{2 \mathrm{R}}=\sum_{\substack{k=1 \\
k \neq 2}}^{n} \frac{D_{(2 S k)}}{D_{(22)}} \widehat{a}_{k}=\frac{D_{(2 S 1)}}{D_{(22)}} \widehat{a}_{1 \mathrm{R}}=S_{21} \widehat{a}_{1 \mathrm{R}}, \\
\widehat{b}_{2 \mathrm{I}}=\sum_{\substack{k=1 \\
k \neq 2}}^{n} \frac{D_{(2 S k)}}{D_{(22)}} \widehat{a}_{k}=\frac{D_{(2 S 4)}}{D_{(22)}} \widehat{a}_{4 \mathrm{I}}=S_{24} \widehat{a}_{4 \mathrm{I}}, \\
\Gamma_{2 \mathrm{R}}^{\prime}=\Gamma_{2 \mathrm{I}}^{\prime}=\frac{D_{(2 S 2)}}{D_{(22)}}=S_{21}^{2} \Gamma_{1}=S_{21}^{2} \Gamma_{\mathrm{A}} e^{-2 \gamma l} .
\end{gathered}
$$

It is defined that the matrix $\mathbf{D}=1-\mathbf{S} \boldsymbol{\Gamma}$, where $\boldsymbol{\Gamma}$ is a diagonal matrix. The elements of $\Gamma$ are the load reflection coefficient on different ports of the DCD. In (15) to (17), $D_{(i S k)}$ is a determinant that the column $i$ of the matrix $\mathbf{D}$ is changed to be the column $k$ of the matrix $\mathbf{S}$. $D_{(i i)}$ is also a determinant that the column $i$ and row $i$ of the matrix $\mathbf{D}$ are deleted. As can be seen from (17), when the DCD is inserted into the interconnected system, the reflection coefficient $\Gamma_{2 \mathrm{R}}^{\prime}$ is equal to $\Gamma_{2 \mathrm{I}}^{\prime}$. It means that the output impedance of the equivalent excitation source is the same under the condition of the radiation and injection test. Hence, the second equivalent condition (i.e., $Z_{\mathrm{SI}}=Z_{\mathrm{SR}}$ ) discussed above can be satisfied.
According to the equivalent circuit model in Figure 9, the radiation response $U_{\mathrm{BR}}$ and the injection response $U_{\mathrm{BI}}$ of the equipment $\mathrm{B}$ can be derived as follows:

$$
\begin{gathered}
U_{\mathrm{BR}}=\frac{\widehat{b}_{2 \mathrm{R}} \sqrt{Z_{\mathrm{C}}}\left(1+\Gamma_{\mathrm{B}}\right)}{1-\Gamma_{2 \mathrm{R}}^{\prime} \Gamma_{\mathrm{B}}}, \\
U_{\mathrm{BI}}=\frac{\widehat{b}_{2 \mathrm{I}} \sqrt{Z_{\mathrm{C}}}\left(1+\Gamma_{\mathrm{B}}\right)}{1-\Gamma_{2 \mathrm{I}}^{\prime} \Gamma_{\mathrm{B}}} .
\end{gathered}
$$

According to the equivalent principle of the two test methods (i.e., $U_{\mathrm{BI}}=U_{\mathrm{BR}}$ ), from (13) to (18), the corresponding relation between the equivalent injection voltage source $U_{\mathrm{SI}}$ and the lumped radiation voltage source $U_{\mathrm{SR}}$ can be derived as follows:

$$
U_{\mathrm{SI}}=S_{21} S_{24}^{-1}\left(1-\Gamma_{\mathrm{A}} e^{-2 \gamma l}\right) U_{\mathrm{SR}} .
$$

If the equipment $\mathrm{A}$ is an antenna or a transmitter, the reflection coefficient $\Gamma_{\mathrm{A}}$ usually remains unchanged. In addition, the $S$ parameter of the DCD is constant. According to (19), the relation between $U_{\mathrm{SI}}$ and $U_{\mathrm{SR}}$ is linear.

From the above analysis process, when the DCD is inserted into the interconnected system, under the condition of unchanged reflection coefficient $\Gamma_{A}$, the relation between the injection voltage $U_{\text {SI }}$ and the radiated field intensity $E$ is always linear. Therefore, the injection voltage source obtained by the linear extrapolation satisfies the first equivalent condition for the radiation and differential-mode current injection test. 


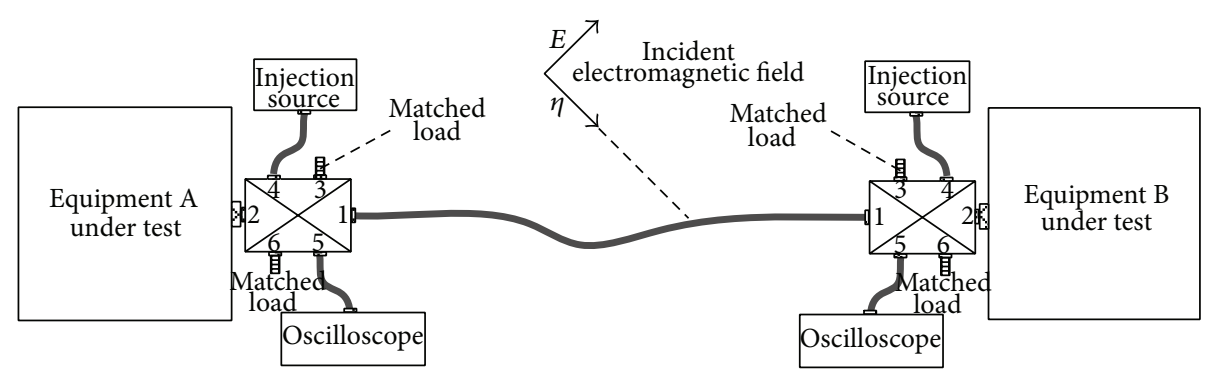

FIgURE 10: The typical test configuration of DDMCI test method.

4.2. Feasibility Analysis on Using the Monitoring Port Response Voltage of the DCD as Equivalent Principle. The equivalent principle for the radiation and injection test in this paper is the equal response voltage on the input port of the equipment B. However, it is very hard for us to directly monitor the input port response voltage in engineering. In order to make the SDMCI method applicable in the practical engineering test, other transmission signals which can be easily monitored should be selected as equivalent principle. The introduction of the DCD solves the problem.

The port 5 of DCD is used to monitor the forward voltage on the transmission line. In this paper, the equal response voltage on port 5 is defined as the equivalent principle for the SDMCI and radiation test. The following article proofs the correctness of using the port 5 response voltage as the equivalent principle. According to the equivalent circuit model in Figure 8, as for the reference plane $T_{5}$, the radiation equivalent source wave $\widehat{b}_{5 \mathrm{R}}$ and the injection equivalent source wave $\widehat{b}_{5 \mathrm{I}}$ that transmit to the direction of impedance $Z_{5}$ can be derived as follows:

$$
\begin{gathered}
\widehat{b}_{5 \mathrm{R}}=\frac{D_{(5 S 1)}}{D_{(55)}} \widehat{a}_{1 \mathrm{R}}=\frac{S_{51}}{1-S_{21}^{2} \Gamma_{1} \Gamma_{\mathrm{B}}} \widehat{a}_{1 \mathrm{R}}, \\
\widehat{b}_{5 \mathrm{I}}=\frac{D_{(5 S 4)}}{D_{(55)}} \widehat{a}_{4 \mathrm{I}}=\frac{S_{54}+S_{21}\left(S_{51} S_{24}-S_{54} S_{21}\right) \Gamma_{1} \Gamma_{\mathrm{B}}}{1-S_{21}^{2} \Gamma_{1} \Gamma_{B}} \widehat{a}_{4 \mathrm{I}} .
\end{gathered}
$$

Assuming that the coupling coefficient of port 4 and port 5 is $m$ and $n$ separately and the unit is $\mathrm{dB}$, then

$$
\begin{gathered}
S_{51} S_{24}=S_{54} S_{21}=\sqrt{\left(1-10^{-m / 10}\right)\left(1-10^{-n / 10}\right) 10^{-(m+n) / 10}}, \\
\widehat{b}_{5 \mathrm{I}}=\frac{\widehat{a}_{4 \mathrm{I}} S_{54}}{1-S_{21}^{2} \Gamma_{1} \Gamma_{\mathrm{B}}} .
\end{gathered}
$$

Under the condition of the radiation and injection test, the response voltages $U_{\mathrm{MR}}$ and $U_{\mathrm{MI}}$ on monitoring port 5 can be derived as follows:

$$
\begin{gathered}
U_{\mathrm{MR}}=\sqrt{Z_{\mathrm{C}}} \widehat{b}_{5 \mathrm{R}}=\frac{S_{51}\left(1-\Gamma_{1}\right) U_{\mathrm{SR}}}{2\left(1-S_{21}^{2} \Gamma_{1} \Gamma_{\mathrm{B}}\right)}, \\
U_{\mathrm{MI}}=\sqrt{Z_{\mathrm{C}} \hat{b}_{5 \mathrm{I}}}=\frac{S_{54} U_{\mathrm{SI}}}{2\left(1-S_{21}^{2} \Gamma_{1} \Gamma_{\mathrm{B}}\right)} .
\end{gathered}
$$

It is assumed that $U_{\mathrm{MR}}=U_{\mathrm{MI}}$; then the relation between $U_{\mathrm{SI}}$ and $U_{\mathrm{SR}}$ can be derived from (22) as follows:

$$
U_{\mathrm{SI}}=S_{21} S_{24}^{-1}\left(1-\Gamma_{\mathrm{A}} e^{-2 \gamma l}\right) U_{\mathrm{SR}}
$$

It can be seen that (23) is equal to (19). It means that the radiation response $U_{\mathrm{BR}}$ is equal to the injection response $U_{\mathrm{BI}}$. Therefore, the above theoretical derivation processes verify the correctness of using the port 5 response voltage as the equivalent principle.

\subsection{Test Procedure of SDMCI}

Step 1. Carry out the low intensity radiation pretest for interconnected systems. The radiated field intensity is selected as $E_{1}$ which can ensure that the response of equipment $\mathrm{B}$ is in the linear region. The output response $U_{\mathrm{MR}}$ on the monitoring port 5 of the DCD is recorded.

Step 2. Obtain the equivalent corresponding relation between injection voltage and radiated electric field intensity. The differential-mode current injection test is carried out through injection port 4 of the DCD. The output response $U_{\mathrm{MI}}$ on the monitoring port 5 is measured at the same time. When $U_{\mathrm{MI}}=U_{\mathrm{MR}}$, the injection voltage $U_{\mathrm{SI}}$ is recorded. The equivalent relation between the injection voltage and the radiated field intensity can be described as $k=U_{\mathrm{SI}} / E_{1}$.

Step 3. Finish the SDMCI test for interconnected systems. If the high intensity radiated field for ultimate examination is $E_{2}$, then the equivalent injection voltage can be calculated by the equation $V_{\mathrm{SI}}=k E_{2}$. Therefore, the SDMCI test can be carried out finally with the equivalent injection voltage $V_{\mathrm{SI}}$ through the injection port 4 of the DCD.

\section{Implementation Technology of DDMCI}

In order to carry out differential-mode current injection test for two types of equipment interconnected by a cable simultaneously, the double differential-mode current injection test (DDMCI) method is proposed in this paper. The test configuration of the DDMCI is shown in Figure 10.

Two directional coupling devices are connected with equipment $\mathrm{A}$ and equipment $\mathrm{B}$ separately. The equivalent 
circuit model is shown in Figure 11, where $U_{\text {SLI }}$ is the injection voltage source of the left DCD and $U_{\text {SRI }}$ is the injection voltage source of the right DCD. Normally, the impedances of equipment $\mathrm{A}$ and equipment $\mathrm{B}$ are not matched. So the responses of equipment $\mathrm{A}$ and equipment $\mathrm{B}$ are the result of the joint action of $U_{\mathrm{SLI}}$ and $U_{\mathrm{SRI}}$.

In order to ensure that the injection response is equal to the radiation response of equipment $\mathrm{A}$ and equipment $\mathrm{B}$, two injection voltage sources $U_{\text {SLI }}$ and $U_{\text {SRI }}$ are required to satisfy certain amplitude and phase relation. The detailed test procedure of DDMCI method is summarized as follows.

Step 1. Carry out low intensity radiation pretest for interconnected system. The radiated electric field intensity is selected as $E_{1}$ which can ensure the responses of equipment $A$ and equipment $\mathrm{B}$ are in the linear region. The amplitude and phase on the left and right monitoring ports of the DCDs are recorded as $U_{5 \mathrm{LR}}, U_{5 \mathrm{RR}}, \varphi_{5 \mathrm{LR}}$, and $\varphi_{5 \mathrm{RR}}$.

Step 2. Obtain the equivalent corresponding relation between injection voltage and radiated electric field intensity. First, the interconnected system is carried out injection test through port $4 \mathrm{~L}$ with the left injection voltage source. The amplitude $U_{5 \mathrm{LI}}$ and phase $\varphi_{5 \mathrm{LI}}$ on the monitoring port $5 \mathrm{~L}$ are measured. When $U_{5 \mathrm{LI}}=U_{5 \mathrm{LR}}$ and $\varphi_{5 \mathrm{LI}}=\varphi_{5 \mathrm{LR}}$, the amplitude and phase of the left injection voltage source remain unchanged. Second, the interconnected system is carried out injection test through port $4 \mathrm{R}$ with the right injection voltage source. The amplitude $U_{5 \mathrm{RI}}$ and phase $\varphi_{5 \mathrm{RI}}$ on the monitoring port $5 \mathrm{R}$ are measured. When $U_{5 \mathrm{RI}}=U_{5 \mathrm{RR}}$ and $\varphi_{5 \mathrm{RI}}=\varphi_{5 \mathrm{RR}}$, the amplitude and phase of the right injection voltage source remain unchanged. Third, repeat the first and the second steps until the conditions of $U_{5 \mathrm{LI}}=U_{5 \mathrm{LR}}, U_{5 \mathrm{RI}}=U_{5 \mathrm{RR}}$, $\varphi_{5 \mathrm{LI}}=\varphi_{5 \mathrm{LR}}$, and $\varphi_{5 \mathrm{RI}}=\varphi_{5 \mathrm{RR}}$ are satisfied simultaneously. Fourth, the amplitudes $U_{\text {SLI }}$ and $U_{\text {SRI }}$ and the phases $\varphi_{\text {SLI }}$ and $\varphi_{\text {SRI }}$ of the two injection voltage sources are recorded finally. The corresponding relation between equivalent injection voltage and radiated electric field intensity can be calculated by $k_{\mathrm{L}}=U_{\mathrm{SLI}} / E_{1}$ and $k_{\mathrm{R}}=U_{\mathrm{SRI}} / E_{1}$.

Step 3. Finish the DDMCI test for interconnected system. If the high intensity radiated field for ultimate examination is $E_{2}$, then the left and right equivalent injection voltage sources can be calculated by $V_{\mathrm{SLI}}=k_{\mathrm{L}} E_{2}$ and $V_{\mathrm{SRI}}=k_{\mathrm{R}} E_{2}$. The phase difference between the two injection voltage sources satisfies $\varphi_{\Delta}=\varphi_{\text {SLI }}-\varphi_{\text {SRI }}$. Finally, the DDMCI test can be carried out through the injection ports $4 \mathrm{~L}$ and $4 \mathrm{R}$ of the DCDs, and it is equivalent to the $E_{2}$ radiation test.

\section{Experimental Verification}

6.1. The Linear Relation Verification between the Equivalent Injection Voltage and the Radiated Field Intensity. The interconnected system under test is a typical nonlinear response system. It is composed of a receiving antenna, a coaxial cable, and RF front-end components. The RF front-end components include a clipping filter, an attenuator, a low noise amplifier (LNA), a sensitivity controller, a directional coupler, and a clipping amplifier. They are integrated together in one container. It is assumed that the receiving antenna is the equipment $A_{1}$ and the container of the RF front-end components is the equipment $\mathrm{B}_{1}$. Because of the nonlinear response characteristic of the clipping filter, LNA, and so on, the above nonlinear response interconnected system is suitable to carry out the verification test.

The single frequency continuous wave radiation test and the differential-mode current injection test are carried out separately for the interconnected system. The radiation and injection configuration diagrams are shown in Figures 12 and 13. The output response of equipment $B_{1}$ is monitored by the spectrum analyzer. The relation curves between the radiated electric field intensity, the injection voltage, and the output response of equipment $\mathrm{B}_{1}$ are recorded separately.

The equal output response of equipment $B_{1}$ for the radiation and injection test is regarded as the equivalent principle. According to the result of the data processing, the relation curves between the equivalent injection voltage, the output response of equipment $\mathrm{B}_{1}$, and the radiated electric field intensity are shown in Figure 14.

It can be seen form Figure 14 that the relation between the radiated electric field intensity and the output response of equipment $B_{1}$ is nonlinear. In contrast, the relation between the radiated electric field intensity and the equivalent injection voltage is linear. In addition, a small number of data points which are in the saturation region do not strictly accord with the linear relation. We believe that it is caused by experimental error. The reason is that the output response of the equipment $B_{1}$ is not sensitive to the radiated field intensity or the injection voltage when the equipment $B_{1}$ works in the saturation region. It means that two different input signals can produce almost the same output response. In conclusion, even for the nonlinear response system, the corresponding relation between the equivalent injection voltage and the radiated electric field intensity at different frequency points is still linear.

6.2. The Validity Verification for SDMCI Test Method. The interconnected system under test is also the above nonlinear response system. The validity verification for SDMCI can be conducted as follows. First, the traditional radiation test is carried out for the interconnected system. The radiation response curve of the equipment $B_{1}$ from the linear region to the saturation region can be obtained. Second, according to the above SDMCI test method, the injection response curve of the equipment $B_{1}$ from the linear region to the saturation region can also be abstained. Third, by calculating and analyzing the output response error of the two test methods, the validity of SDMCI method can be verified.

The response curves of the equipment $\mathrm{B}_{1}$ under the condition of radiation and the SDMCI test at the frequency point of $3.3 \mathrm{GHz}, 4.0 \mathrm{GHz}$ and $5.6 \mathrm{GHz}$ are shown in Figure 15. It can be seen that the output response curve obtained by the SDMCI test is almost exactly the same as that obtained by the radiation test. In order to analyze the error of the SDMCI test method, the test data in Figure 15 are processed. The output response relative error $\eta$ for the SDMCI test method is shown in Table 1. As can be seen from Table 1, the relative error $\eta$ is 


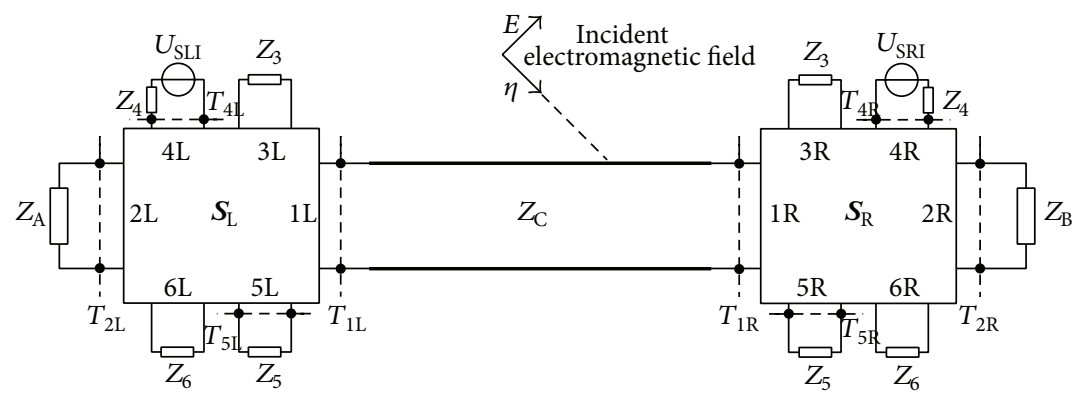

FIGURE 11: The equivalent circuit model of DDMCI test method.
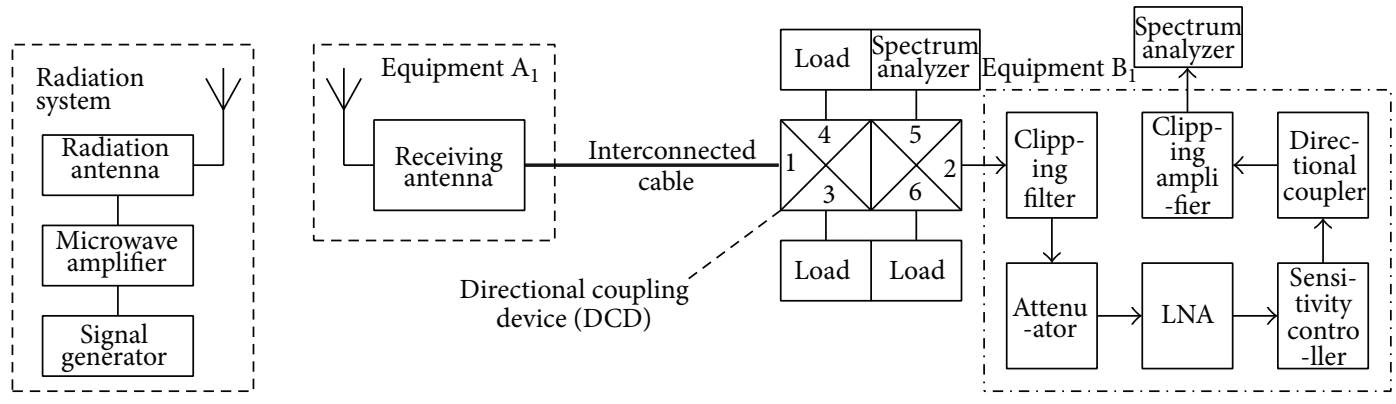

FIGURE 12: The configuration diagram for the radiation test.

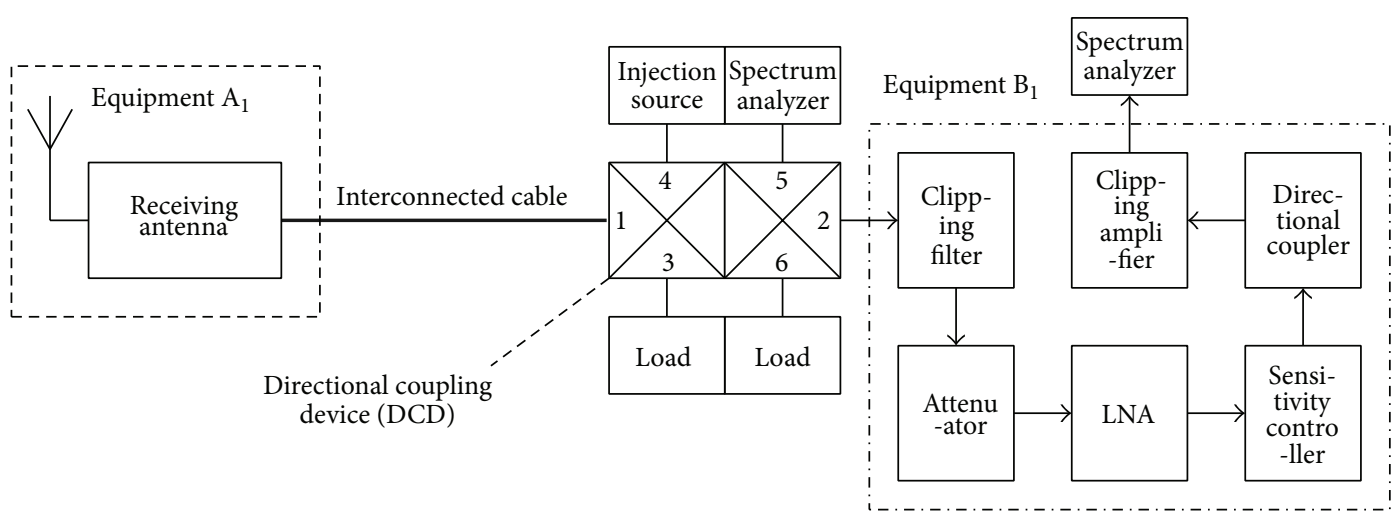

FIGURE 13: The configuration diagram for the differential-mode current injection test.

small and the biggest one is $2.39 \%$. The error comes from the performance fluctuation of the RF front-end components, the noise of active device, the reading error of instrument, and so on. There is no systematic error for the SDMCI test method.

6.3. The Validity Verification for DDMCI Test Method. Two satellite-borne RF front-end low-noise amplifier modules are selected as equipment $A_{2}$ and equipment $B_{2}$ which are connected by a coaxial cable. It is important to note that this kind of interconnected system does not exist in engineering.
The designed interconnected system is only for experimental verification in the extreme condition.

The radiation test configuration is shown in Figure 16. The coaxial cable under test is placed in the shielded room. It is connected with port 1 of the DCD through the interface board. Port 2 of the DCD is connected with the equipment under test. The output port of the equipment is connected with spectrum analyzer (SA) which is used to monitor the output response of the equipment. Outside the shielded room, vector network analyzer (VNA) is used as transmitter and receiver. Port 1 of the VNA is used as signal generator. It is connected with radiation antenna in the shielded room 


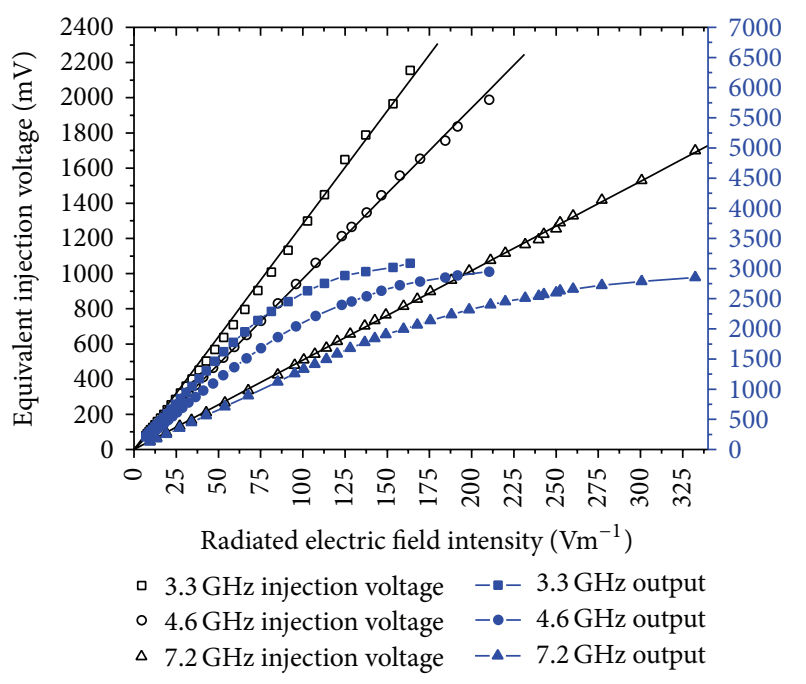

FIGURE 14: The relation curves between the equivalent injection voltage and the radiated electric field intensity.

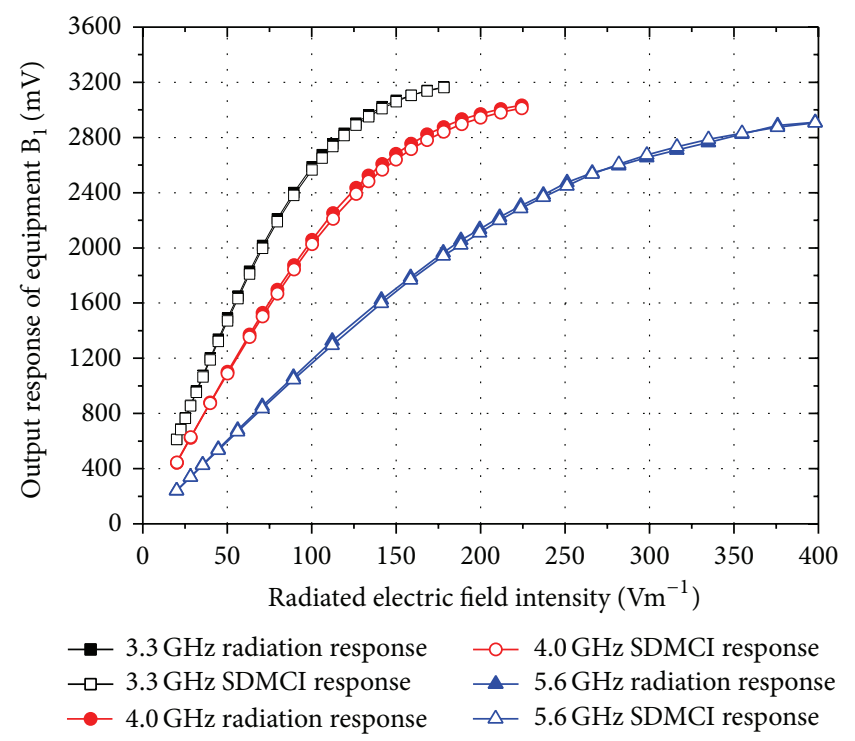

FIGURE 15: The response curves under the condition of radiation and SDMCI test.

through power amplifier. The radiation antenna is placed ahead of the coaxial cable under test. Port 2 of the VNA is used as receiver. It is connected with the monitoring port of the DCD. The amplitude and phase difference of the two monitoring ports of the DCDs can be obtained by testing the parameter $S_{21}$.

The DDMCI test configuration is shown in Figure 17. Port 1 of the VNA is used as injection signal source. In order to carry out differential-mode current injection test for the two interconnected types of equipment simultaneously, the injection signal is divided into two circuits by the power divider. They are connected with the injection ports $4 \mathrm{~L}$ and $4 \mathrm{R}$ of the DCDs separately. In order to ensure that the two
TABLE 1: Output response relative error for SDMCI test method.

\begin{tabular}{lcccccc}
\hline \multirow{2}{*}{ Number } & \multicolumn{2}{c}{$f=3.3 \mathrm{GHz}$} & \multicolumn{2}{c}{$f=4.0 \mathrm{GHz}$} & \multicolumn{2}{c}{$f=5.6 \mathrm{GHz}$} \\
& $E / \mathrm{Vm}^{-1}$ & $\eta / \%$ & $E / \mathrm{Vm}^{-1}$ & $\eta / \%$ & $E / \mathrm{Vm}^{-1}$ & $\eta / \%$ \\
\hline 1 & 20.00 & 0.12 & 20.00 & 0.93 & 19.95 & 0.12 \\
2 & 22.44 & 0.34 & 28.25 & 0.12 & 28.18 & 0.34 \\
3 & 25.18 & 0.46 & 39.91 & 0.34 & 35.48 & 0.69 \\
4 & 28.25 & 0.46 & 50.24 & 1.14 & 44.67 & 1.49 \\
5 & 31.70 & 1.14 & 63.25 & 1.37 & 56.23 & 1.71 \\
6 & 35.57 & 1.26 & 70.96 & 1.60 & 70.79 & 1.94 \\
7 & 39.91 & 1.14 & 79.62 & 1.83 & 89.13 & 1.83 \\
8 & 44.77 & 1.26 & 89.34 & 1.60 & 112.20 & 2.39 \\
9 & 50.24 & 1.37 & 100.24 & 1.60 & 141.25 & 1.49 \\
10 & 56.37 & 1.14 & 112.47 & 1.83 & 158.49 & 1.03 \\
11 & 63.25 & 1.14 & 126.19 & 1.83 & 177.83 & 1.14 \\
12 & 70.96 & 1.03 & 133.67 & 1.71 & 188.36 & 1.60 \\
13 & 79.62 & 0.92 & 141.59 & 1.60 & 199.53 & 1.14 \\
14 & 89.34 & 0.92 & 149.98 & 1.60 & 211.35 & 1.03 \\
15 & 100.24 & 0.92 & 158.87 & 1.37 & 223.87 & 0.80 \\
16 & 106.18 & 0.92 & 168.28 & 1.37 & 237.14 & 0.69 \\
17 & 112.47 & 0.69 & 178.25 & 1.26 & 251.19 & 1.03 \\
18 & 119.13 & 0.57 & 188.81 & 1.14 & 266.07 & 0.34 \\
19 & 126.19 & 0.46 & 200.00 & 1.03 & 281.84 & 0.35 \\
20 & 133.67 & 0.46 & 211.85 & 0.80 & 298.54 & 0.69 \\
21 & 141.59 & 0.46 & 224.40 & 0.69 & 316.23 & 0.81 \\
22 & 149.98 & 0.34 & - & - & 334.97 & 0.81 \\
23 & 158.87 & 0.00 & - & - & 354.81 & 0.23 \\
24 & 168.28 & 0.12 & - & - & 375.84 & 0.46 \\
25 & 178.25 & 0.12 & - & - & 398.11 & 0.12 \\
\hline & & & & & &
\end{tabular}

circuits have different amplitude and phase, the continuously adjustable attenuator and $360^{\circ} / \mathrm{GHz}$ phase shifter are inserted into one circuit. Port 2 of the VNA is also used as receiver. It is used to measure the response on the monitoring ports $5 \mathrm{~L}$ and $5 \mathrm{R}$ of the DCDs under the condition of DDMCI test. The other test configuration is the same as in the above radiation test.

According to the test configuration in Figures 16 and 17 and the above test procedure, in the working frequency band of the low-noise amplifier modules, the three frequency points $1.510 \mathrm{GHz}, 1.605 \mathrm{GHz}$, and $1.75 \mathrm{GHz}$ are selected to carry out radiation and DDMCI test. The radiation and DDMCI response curve is shown in Figures 18 and 19.

As can be seen from Figures 18 and 19, the output response curve of equipment $A_{2}$ and equipment $B_{2}$ obtained by DDMCI test is almost exactly the same as that obtained by radiation test. In order to analyze the error of DDMCI test method, the test data in Figures 18 and 19 are processed. The output response relative error for DDMCI test method is shown in Table 2. As can be seen from Table 2, the biggest output response relative error is only $3.39 \%$. The test results indicate that the DDMCI test method can effectively simulate the HIRF effect experiment for nonlinear interconnected system. 
TABLE 2: Output response relative error for DDMCI test method.

\begin{tabular}{lccccccccc}
\hline \multirow{2}{*}{ Number } & \multicolumn{3}{c}{$f=1.510 \mathrm{GHz}$} & \multicolumn{3}{c}{$f=1.605 \mathrm{GHz}$} & \multicolumn{3}{c}{$f=1.750 \mathrm{GHz}$} \\
& $E / \mathrm{Vm}^{-1}$ & $\eta_{\mathrm{A} 2} / \%$ & $\eta_{\mathrm{B} 2} / \%$ & $E / \mathrm{Vm}^{-1}$ & $\eta_{\mathrm{A} 2} / \%$ & $\eta_{\mathrm{B} 2} / \%$ & $E / \mathrm{Vm}^{-1}$ & $\eta_{\mathrm{A} 2} / \%$ & $\eta_{\mathrm{B} 2} / \%$ \\
\hline 1 & 12.62 & 0.35 & 0.35 & 14.16 & 0.34 & 0.92 & 12.62 & 1.49 \\
2 & 15.89 & 0.00 & 0.23 & 17.83 & 0.00 & 1.26 & 15.89 & 1.26 \\
3 & 20.00 & 1.14 & 1.49 & 22.44 & 0.46 & 0.57 & 20.00 & 0.57 & 2.61 \\
4 & 25.18 & 0.80 & 1.26 & 28.25 & 1.16 & 0.12 & 22.44 & 0.81 \\
5 & 31.70 & 0.46 & 1.26 & 35.57 & 1.39 & 0.69 & 25.18 & 0.69 \\
6 & 39.91 & 0.34 & 0.92 & 44.77 & 1.62 & 1.16 & 28.25 & 1.51 \\
7 & 44.77 & 0.12 & 0.69 & 50.23 & 1.86 & 1.16 & 31.70 & 1.03 \\
8 & 50.24 & 0.23 & 0.57 & 56.37 & 1.86 & 1.39 & 35.57 & 0.69 \\
9 & 56.37 & 0.35 & 0.34 & 63.25 & 1.98 & 1.51 & 39.91 & 0.46 \\
10 & 63.25 & 0.46 & 0.46 & 70.96 & 1.86 & 1.62 & 44.77 & 1.16 \\
11 & 66.99 & 0.81 & 0.12 & 75.17 & 1.98 & 1.51 & 50.24 & 1.51 \\
12 & 70.96 & 0.35 & 0.34 & 79.62 & 1.74 & 1.62 & 56.37 & 0.23 \\
13 & 75.16 & 0.93 & 0.23 & 84.34 & 1.39 & 1.27 & 59.71 & 0.23 \\
14 & 79.62 & 0.35 & 0.12 & 89.34 & 1.04 & 1.39 & 63.25 & 0.00 \\
15 & 84.34 & 0.81 & 0.35 & - & - & - & 66.99 & 2.21 \\
16 & 89.34 & 0.23 & 0.00 & - & - & - & 70.96 & 0.23 \\
\hline
\end{tabular}

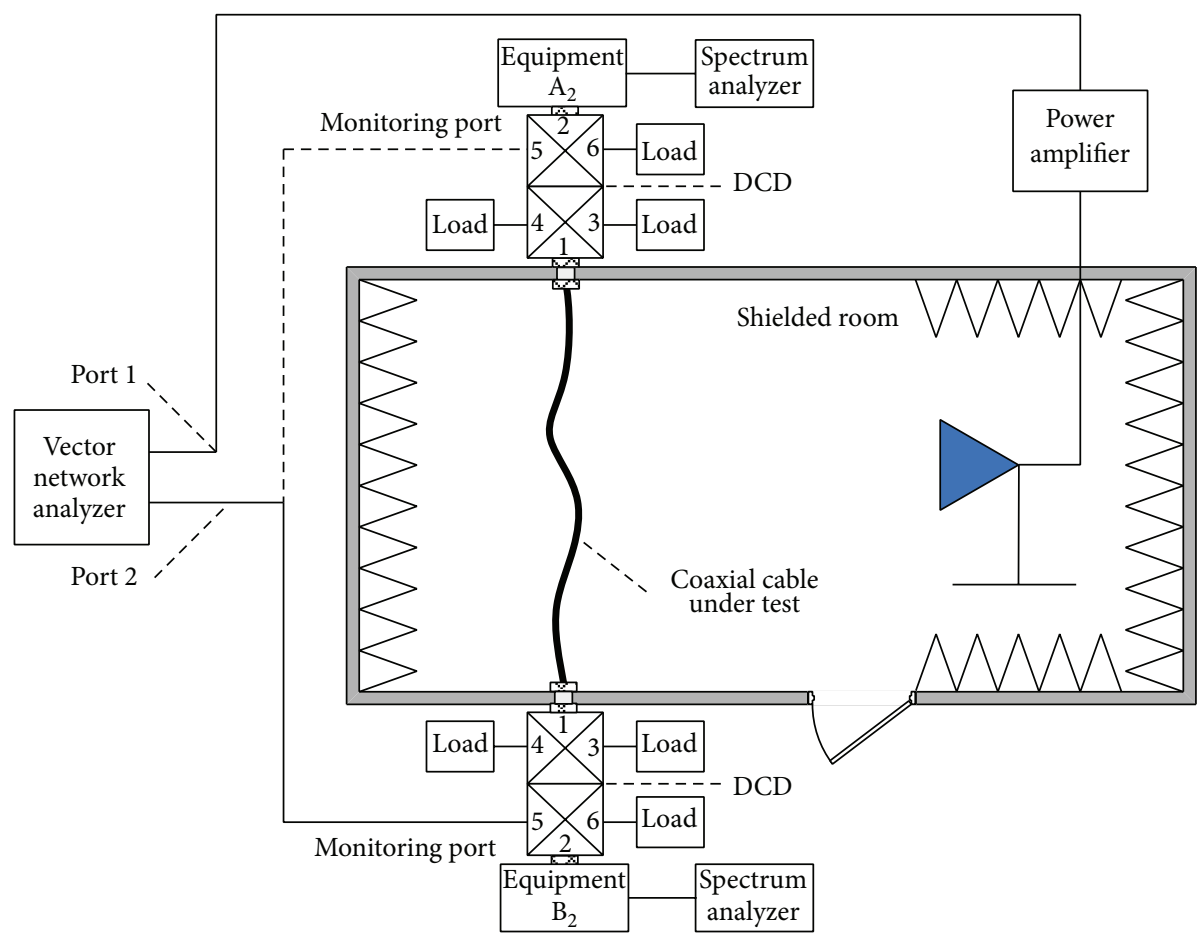

FIGURE 16: The configuration diagram for the radiation test.

\section{Conclusions}

The theory, model, method, and implementation technique of the wideband differential-mode current injection test technology are systematically studied in this paper. The injection and radiation response analysis model and the injection voltage source extrapolation model in HIRF are established. The conditions of using injection as a substitute for radiation are confirmed. The equivalent injection voltage source can be obtained by the linear extrapolation. The function and structure design scheme of the directional coupling device (DCD) is proposed. The forward voltage extraction, interference signal injection, and normal transmission signal monitoring in the interconnected system are realized with the support of DCD. On the basis of the above research, the SDMCI test method and the DDMCI test method based on the DCD are summarized. The typical nonlinear response systems are selected as EUT. The test results indicate that 


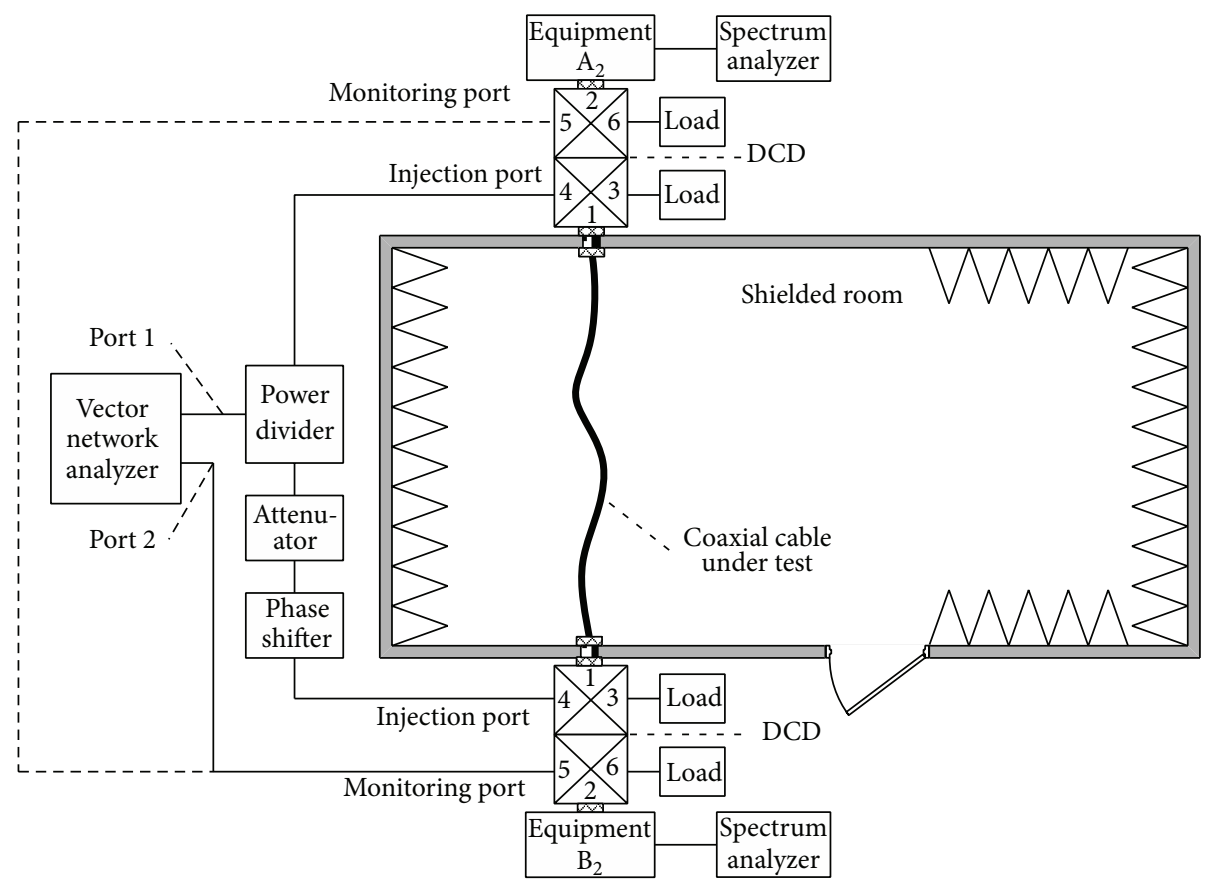

FIGURE 17: The configuration diagram for the DDMCI test.

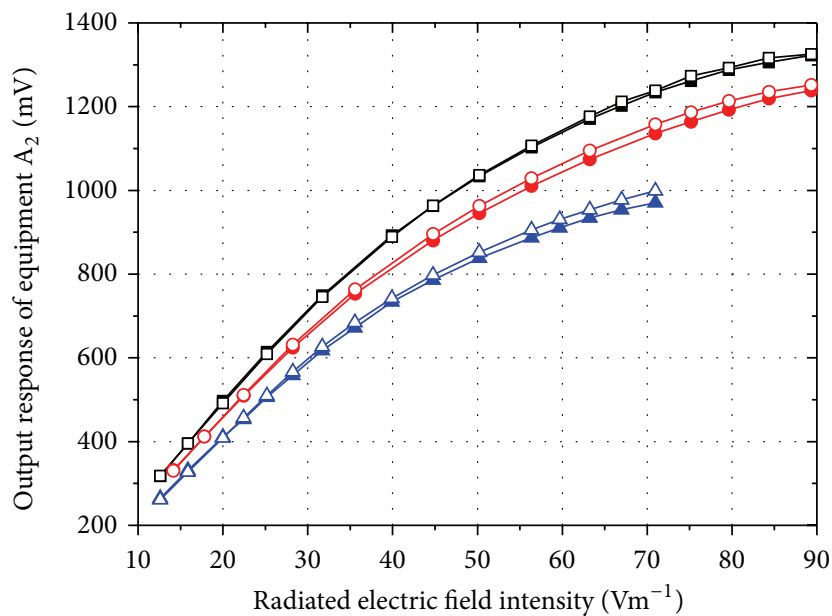

$1.510 \mathrm{GHz}$ radiation response $-0-1.605 \mathrm{GHz}$ DDMCI response $\neg-1.510 \mathrm{GHz}$ DDMCI response $\quad-1.750 \mathrm{GHz}$ radiation response - $1.605 \mathrm{GHz}$ radiation response $\triangle-1.750 \mathrm{GHz}$ DDMCI response

FIGURE 18: The radiation and DDMCI response curve of equipment $\mathrm{A}_{2}$.

the biggest output response relative error is smaller than $5 \%$. They verify the validity of the SDMCI and the DDMCI test methods.

\section{Conflict of Interests}

The authors declare that there is no conflict of interests regarding the publication of this paper.

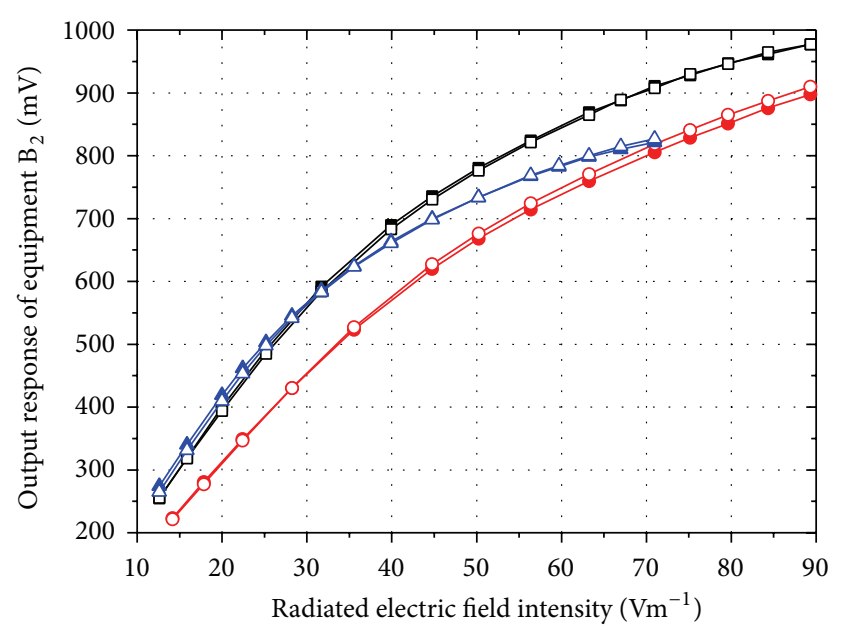

- $1.510 \mathrm{GHz}$ radiation response $-0-1.605 \mathrm{GHz}$ DDMCI response

$\neg-1.510 \mathrm{GHz}$ DDMCI response $\_1.750 \mathrm{GHz}$ radiation response

$-1.605 \mathrm{GHz}$ radiation response $\triangle-1.750 \mathrm{GHz}$ DDMCI response

FIgURE 19: The radiation and DDMCI response curve of equipment $\mathrm{B}_{2}$.

\section{Acknowledgments}

This work is supported by the National Natural Science Foundation of China under Grant 61372040 and the Arm Preresearch Program under Grant 51333040101.

\section{References}

[1] N. J. Carter and E. G. Stevens, "Bulk current injection (BCI): Its past, present and future in aerospace," in Proceedings of the 
IEE Colloquium on EMC Testing for Conducted Mechanisms, pp. 2/1-2/12, May 1996.

[2] G. A. Rasek and M. Gabrisak, "Wire bundle currents for high intensity radiated fields (HIRF) and indirect effects of lightning (IEL) with focus on bulk current injection (BCI) test," in Proceedings of the 21st International Conference Radioelektronika, pp. 1-10, Brno, Czech Republic, April 2011.

[3] J. W. Adams, J. Cruz, and D. Melquist, "Comparison measurements of currents induced by radiation and injection," IEEE Transactions on Electromagnetic Compatibility, vol. 34, no. 3, pp. 360-362, 1992.

[4] J. Perini and L. S. Cohen, "On the equivalence of radiated and injected tests", in Proceedings of the IEEE International Symposium on Electromagnetic Compatibility, pp. 77-80, August 1995.

[5] D. H. Trout, "Investigation of the bulk current injection technique by comparison to induced currents from radiated electromagnetic fields," in Proceedings of the IEEE International Symposium on Electromagnetic Compatibility, pp. 412-417, Santa Clara, Calif, USA, August 1996.

[6] S. Pignari and F. G. Canavero, "On the equivalence between radiation and injection in $\mathrm{BCI}$ testing," in Proceedings of the IEEE International Symposium on Electromagnetic Compatibility (EMC '97), pp. 179-182, May 1997.

[7] G. Cerri, R. de Leo, V. M. Primiani, S. Pennesi, and P. Russo, "Wide-band characterization of current probes," IEEE Transactions on Electromagnetic Compatibility, vol. 45, no. 4, pp. 616-625, 2003.

[8] F. Grassi, F. Marliani, and S. A. Pignari, "Circuit modeling of injection probes for bulk current injection," IEEE Transactions on Electromagnetic Compatibility, vol. 49, no. 3, pp. 563-576, 2007.

[9] S. Pignari, F. Grassi, F. Marliani, and F. G. Canavero, "Experimental characterization of injection probes for bulk current injection," in Proceedings of the 28th General Assemblies International Union of Radio Science (URSI '05), Paper EA.4 (0494), New Delhi, India, October 2005.

[10] S. Pignari and F. G. Canavero, "Theoretical assessment of bulk current injection versus radiation," IEEE Transactions on Electromagnetic Compatibility, vol. 38, no. 3, pp. 469-477, 1996.

[11] D. H. Trout and N. F. Audeh, "Evaluation of electromagnetic radiated susceptibility testing using induced current," in Proceedings of the IEEE Aerospace Conference, vol. 4, pp. 69-80, Aspen, Colo, USA, February 1997.

[12] S. Caniggia and F. Maradei, "SPICE-like models for the analysis of the conducted and radiated immunity of shielded cables," IEEE Transactions on Electromagnetic Compatibility, vol. 46, no. 4, pp. 606-616, 2004.

[13] A. Orlandi, "Circuit model for bulk current injection test on shielded coaxial cables," IEEE Transactions on Electromagnetic Compatibility, vol. 45, no. 4, pp. 602-615, 2003.

[14] A. Orlandi, G. Antonini, and R. M. Rizzi, "Equivalent circuit model of a bundle of cables for Bulk Current Injection (BCI) test," IEEE Transactions on Electromagnetic Compatibility, vol. 48, no. 4, pp. 701-713, 2006.

[15] J. Perini, "Radiated versus injected measurements: when are they equivalent?" in Proceedings of the 10th International Zurich Symposium on Electromagnetic Compatibility, pp. 343-348, 1993.

[16] J. Perini and L. S. Cohen, "Radiated and injected tests: when are they equivalent?" in Proceedings of the 11th International
Zurich Symposium on Electromagnetic Compatibility, pp. 231235, March 1995.

[17] M. Klingler, M. Szelag, and M. Heddebaut, "Double bulk current injection: a possible substitute to field to wire coupling," in Proceedings of the International Symposium in Electromagnetic Environments Consequences (EUROEM '94), pp. 1249-1256, Bordeaux, France, 1994.

[18] F. Grassi, G. Spadacini, F. Marliani, and S. A. Pignari, "Use of double bulk current injection for susceptibility testing of avionics," IEEE Transactions on Electromagnetic Compatibility, vol. 50, no. 3, pp. 524-535, 2008.

[19] G. Spadacini and S. A. Pignari, "A bulk current injection test conforming to statistical properties of radiation-induced effects," IEEE Transactions on Electromagnetic Compatibility, vol. 46, no. 3, pp. 446-458, 2004.

[20] F. M. Tesche, M. V. lanoz, and T. Karlsson, EMC Analysis Methods and Computational Models, John Wiley \& Sons, New York, NY, USA, 1997.

[21] C. E. Baum, "Generalization of the BLT equation," in Proceedings of 13th International Zurich Symposium on Electromagnetic Compatibility, pp. 131-136, March 1999.

[22] F. M. Tesche, "Development and use of the BLT equation in the time domain as applied to a coaxial cable," IEEE Transactions on Electromagnetic Compatibility, vol. 49, no. 1, pp. 3-11, 2007.

[23] D. M. Pozar, Microwave Engineering, John Wiley \& Sons, New York, NY, USA, 4th edition, 2011.

[24] Z. Q. Li, J. Z. She, and B. X. Gao, Foundation for Microwave Engineering, Tsinghua University Press, Beijing, China, 2004. 

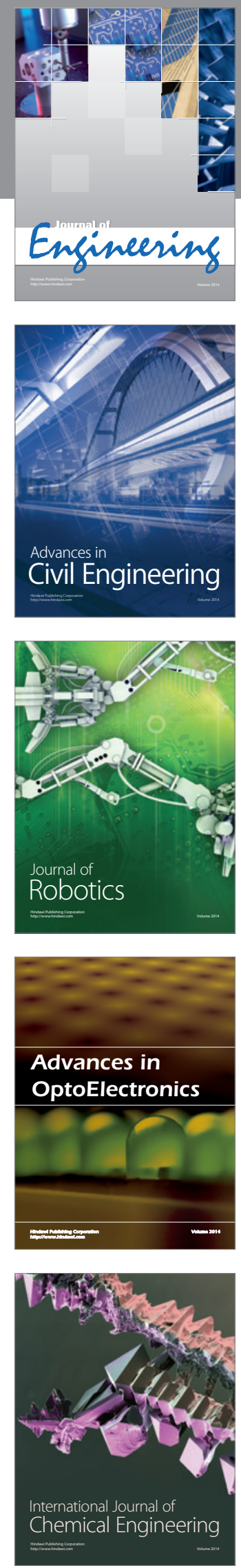

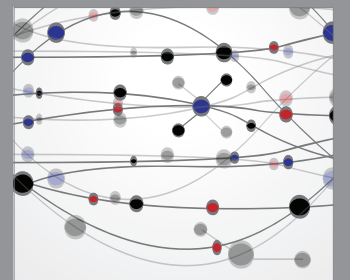

The Scientific World Journal
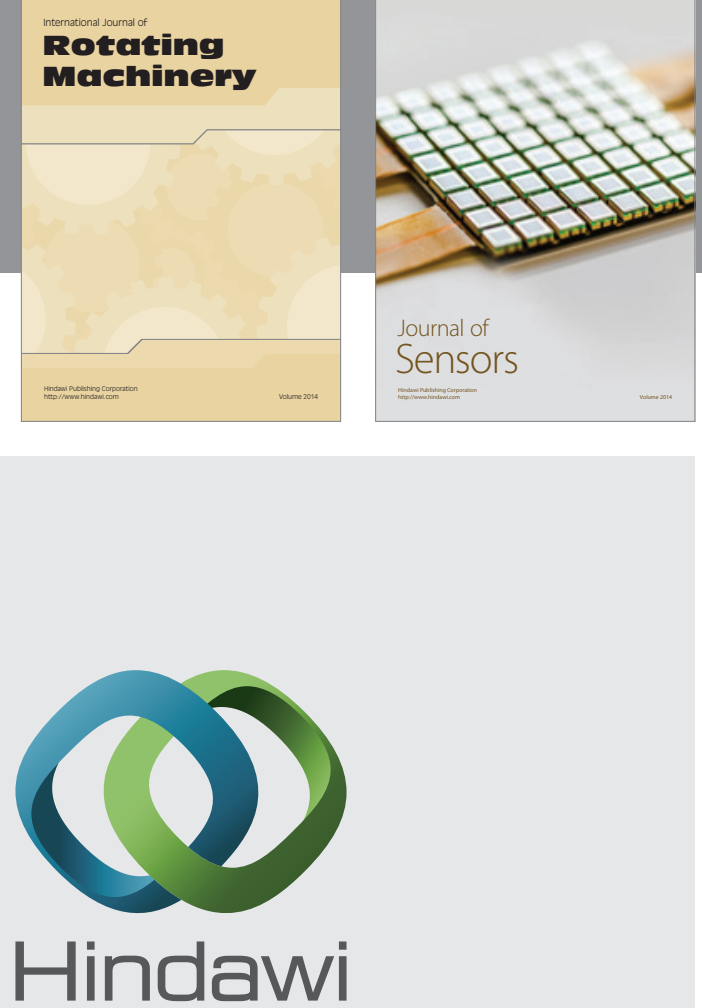

Submit your manuscripts at http://www.hindawi.com
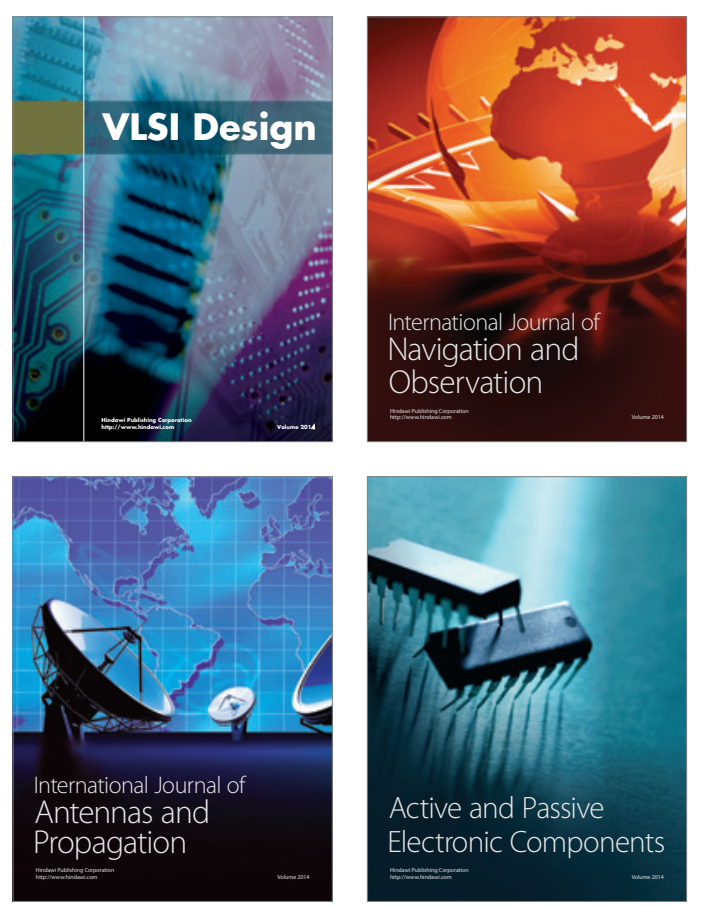
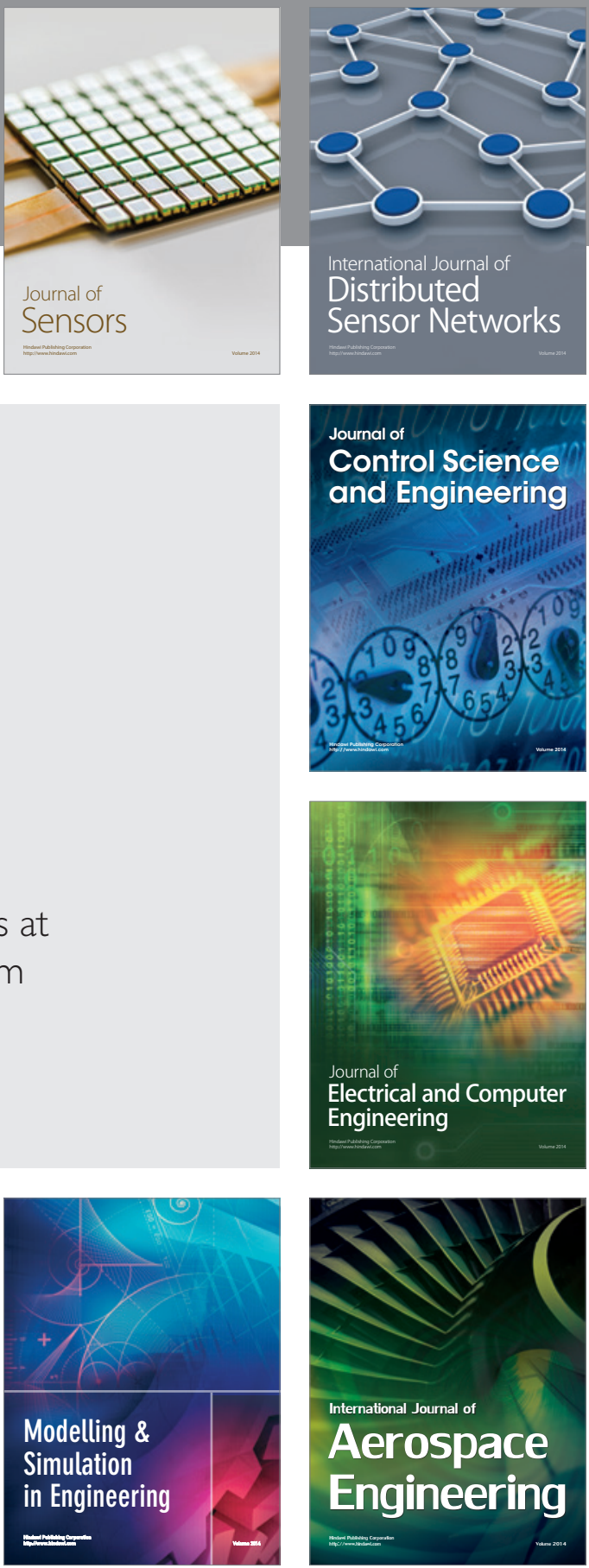

Journal of

Control Science

and Engineering
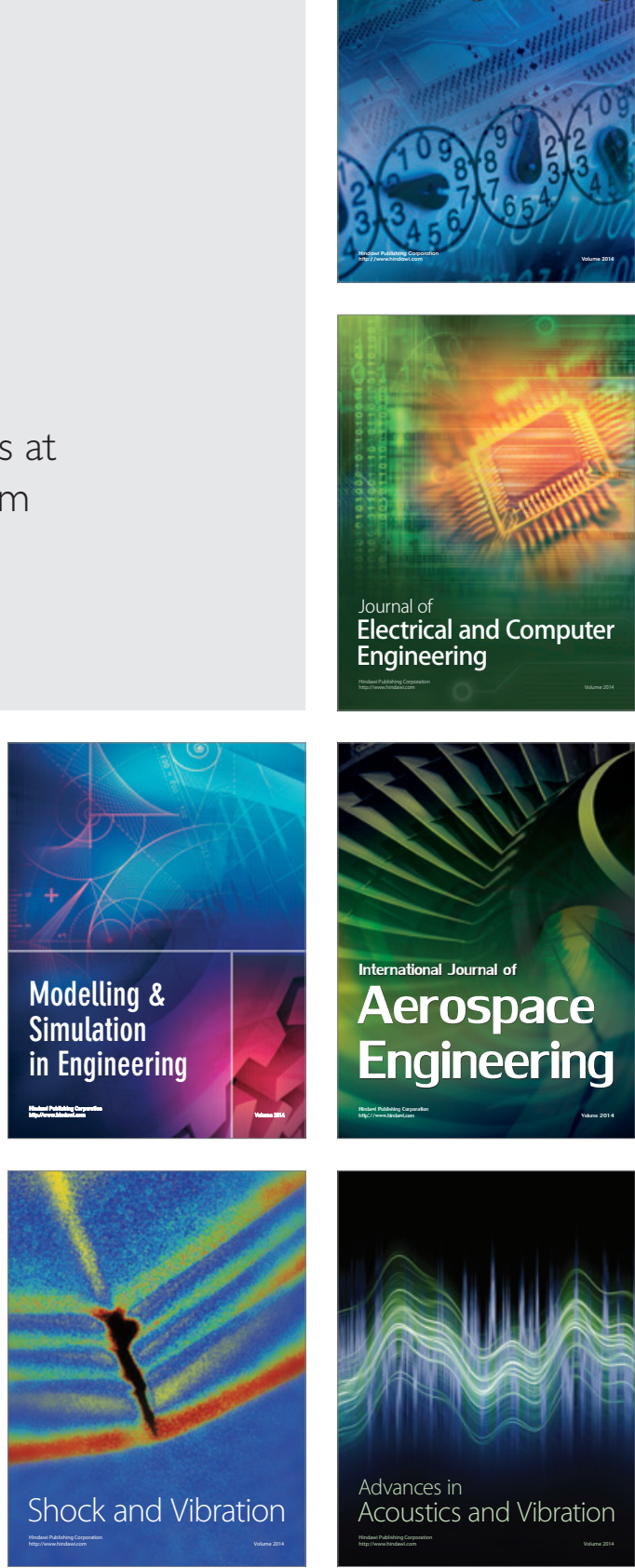